\title{
Extensive cavo-atrial and hepatic venous tumor thrombus in a mismanaged retroperitoneal pediatric germ cell tumor: A unique surgical challenge
}

\author{
Mufaddal Kazi', Pradeep Kaushik², Shivpal Saini', Dhiraj Premchandani', Sajid Shafique Qureshi' ${ }^{1,3}$ \\ 'Division of Paediatric Surgical Oncology, Department of Surgical Oncology, \\ Tata Memorial Hospital and Advanced Centre for Training Research and Education in Cancer (ACTREC), Tata Memorial Centre, Mumbai, India, \\ ${ }^{2}$ Department of Paediatric Cardiac Surgery, SRCC Children's Hospital-Narayana Health, Mumbai, India, \\ ${ }^{3}$ Homi Bhabha National Institute (HBNI), Mumbai, India
}

\begin{abstract}
Retroperitoneal germ cell tumor with tumor thrombosis of the inferior vena cava (IVC) represents a rare phenomenon. Its extension to the hepatic veins (HVs) has not been reported yet. In the present case, a 30-month-old girl had a recurrent retroperitoneal yolk sac tumor with liver metastasis. In addition, there were tumor thrombi in the IVC, right atrium (RA), and all three HVs. The child was operated after a satisfactory response to chemotherapy. Excision of the retroperitoneal tumor with right hepatectomy, retrohepatic caval resection, $\mathrm{HV}$, and RA thrombectomy was performed under a cardiac bypass. HV cloaca was patched with pericardium while the IVC resection was tolerated without reconstruction. Her postoperative recovery was normal. The child currently remains disease-free and symptom-free at 12 months. This case demonstrates the technique of hepatic venous thrombectomy and the feasibility of radical surgery even for a metastatic disease when tumor biology is favorable. Despite wide-spread tumor thrombosis that would have been deemed unresectable, complete resections are possible in referral centers.
\end{abstract}

Key Words: Germ cell neoplasm; Hepatic veins; Tumor thrombosis; Inferior vena cava

\section{INTRODUCTION}

Primary retroperitoneal germ cell tumors (GCT) account for fewer than $4 \%$ of all GCTs [1]. Patients with extensive involvement of the inferior vena cava (IVC) by vascular thrombus or infiltration requiring vessel resection are unusual in GCTs. Such tumor thrombus extension into the hepatic veins (HV) has not been reported yet. Here we report a case of misman-

Received: March 6, 2021, Accepted: March 30, 2021

Corresponding author: Sajid Shafique Qureshi

Division of Paediatric Surgical Oncology, Department of Surgical Oncology,

Tata Memorial Hospital and Advanced Centre for Training Research and

Education in Cancer (ACTREC), Tata Memorial Centre, Ernest Borges Road,

Parel, Mumbai 400012, India

Tel: +91-22-24177276, Fax: +91-22-24146937,

E-mail: sajidshafiques@hotmail.com

ORCID: https://orcid.org/0000-0002-6770-5887

Copyright (C) The Korean Association of Hepato-Biliary-Pancreatic Surgery aged recurrent pediatric GCT with tumor thrombus in the retrohepatic IVC extending to the right atrium (RA) along with tumor thrombi in all three major HVs managed surgically.

\section{CASE}

A 30-month-old girl with a right suprarenal mass was being treated on the lines of neuroblastoma without tissue diagnosis at another center. The child received two cycles of carboplatin and etoposide following which right adrenalectomy was performed. Histopathology found no viable tumor cells. After a disease-free interval of one year, the child presented at our institution with difficulty in breathing and abdominal distension. Computed tomography (CT) revealed a $6-\mathrm{cm}$ necrotic mass in the right supra-renal area with infiltration of the liver, separate liver metastasis in the right hemi-liver (Fig. 1A), thrombus in the HV, IVC, and the RA (Fig. 1B, 1C). Ascites and pleural effusion were also present. The child was stabilized and a biopsy performed was suggestive of a yolk sac tumor. Alpha-fetoprotein (AFP) level was 54,000 ng/mL. After three 
cycles of Cisplatin, Etoposide, and Bleomycin (PEB), there was marked symptomatic improvement along with log reductions in AFP level to $376 \mathrm{ng} / \mathrm{mL}$. Radiological reduction in the size of the mass was equally evident along with disappearance of ascites and pleural effusion. However, there were persistent thromboses of HVs and IVC with a reduction in the thrombus within the RA (Fig. 1D). The entire liver exhibited congestive changes on contrast CT with filling defects in the short hepatic and caudate veins. No portal collaterals were seen. The IVC thrombus extended just above the renal veins inferiorly and to the RA superiorly. Liver metastasis noted in segments 6, 7, and 8 had reduced in size. Doppler ultrasound confirmed the thrombi in the HVs with partial flow along with incomplete recanalization of the thrombus in the IVC. Portal flows were antegrade without significant collaterals. In view of serological and morphological responses of the tumor sites to chemotherapy, the patient was planned for a surgical resection.

Tumor excision with right hepatectomy and cavo-atrial and hepatic venous thrombectomy was planned under a cardiac bypass. A bilateral subcostal incision was undertaken It was extended vertically for sternotomy. Extensive adhesions from the previous surgery were cleared. The retroperitoneal mass was detected to be densely adherent to the inferior surface of the right hemiliver, the diaphragm, and the right renal hilum. The liver was deeply congested and cyanosed (Fig. 2A). IVC was found to be filled with thrombus to an extent that mirrored radiologic findings. Large lumbar veins, phrenic veins, and falciform veins were found along with a dilated azygous system in the chest. IVC just above the renal veins was test clamped to observe hemodynamic instability. None was found. The mass along with the right liver was mobilized. The involved diaphragm was excised. The right Glissonian pedicle was staple transected en-masse. Parenchymal transection for right hepatectomy was performed until only the right hepatic vein (RHV) remained as an attachment (Fig. 2B). Retrograde intraparenchymal dissection of the RHV was performed until a tumor-free area was found at which point the RHV was stapled and the specimen was removed. At this point, the ascending aorta and the superior vena cava were cannulated and a hypothermic cardiac bypass was initiated without cardioplegia. The IVC was opened including the RA. Blood that was suctioned was returned to the pump for reperfusion to reduce blood loss. Thrombectomy from the cava was performed successfully with sequential hepatic venous thrombectomy from the caval hepatic venous ostia (Fig. 2B). All hepatic venous thrombi were removed with direct traction. Clearance was confirmed using intraoperative ultrasound as well as dramatic regression of the hepatic congestion visible grossly (Fig. 2C). The tumor thrombus was densely adherent to the endocardium. A small partial thickness of the RA was excised. Since the primary tumor mass was also adherent to the IVC, the portion of the retrohepatic IVC was also removed. Prior test clamp of the IVC above the renal hilum demonstrated no hemodynamic compromise along with abundant collaterals in the retroperitoneum. Thus, no reconstruction of the IVC was considered. RA was closed primarily. An autologous pericardium was used to patch the portion of IVC at the hepatic cloaca to drain the common trunk of the middle and left HVs (Fig. 2D). The diaphragm was closed
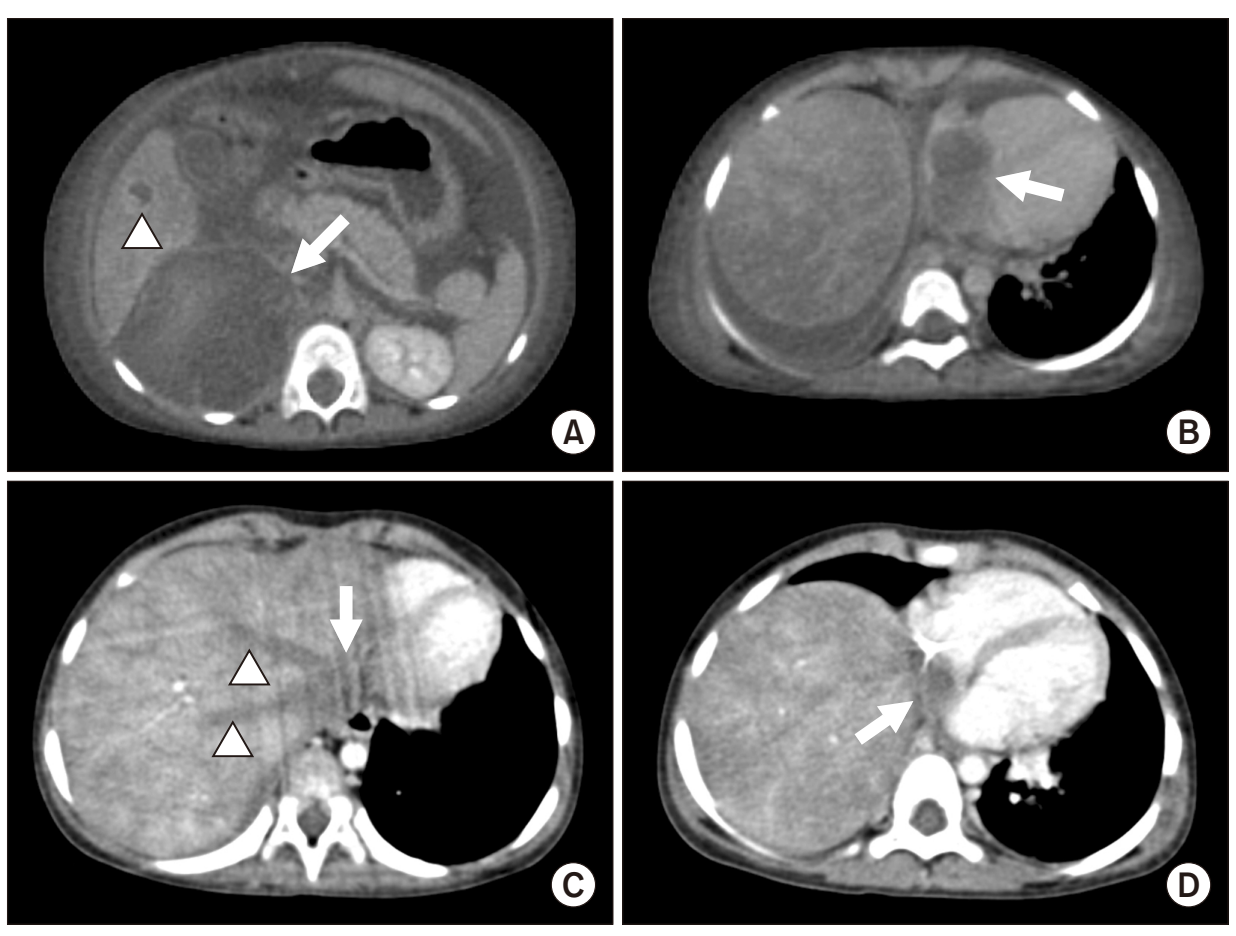

Fig. 1. (A) Pre-chemotherapy computed tomography $(\mathrm{CT})$ demonstrating a large right retroperitoneal mass (arrow) with ascites and liver metastasis (arrowhead). (B) $\mathrm{CT}$ showing thrombus filling the right atrium (arrow) with congested appearance of the liver and ascites. (C) Post-chemotherapy CT with persistent thrombosis of the hepatic veins (arrowheads) and a thrombus filling and expanding the retrohepatic inferior vena cava (arrow). (D) Reduction in size of the right atrial thrombus (arrow) and some resolution of congestion of the liver after chemotherapy. 

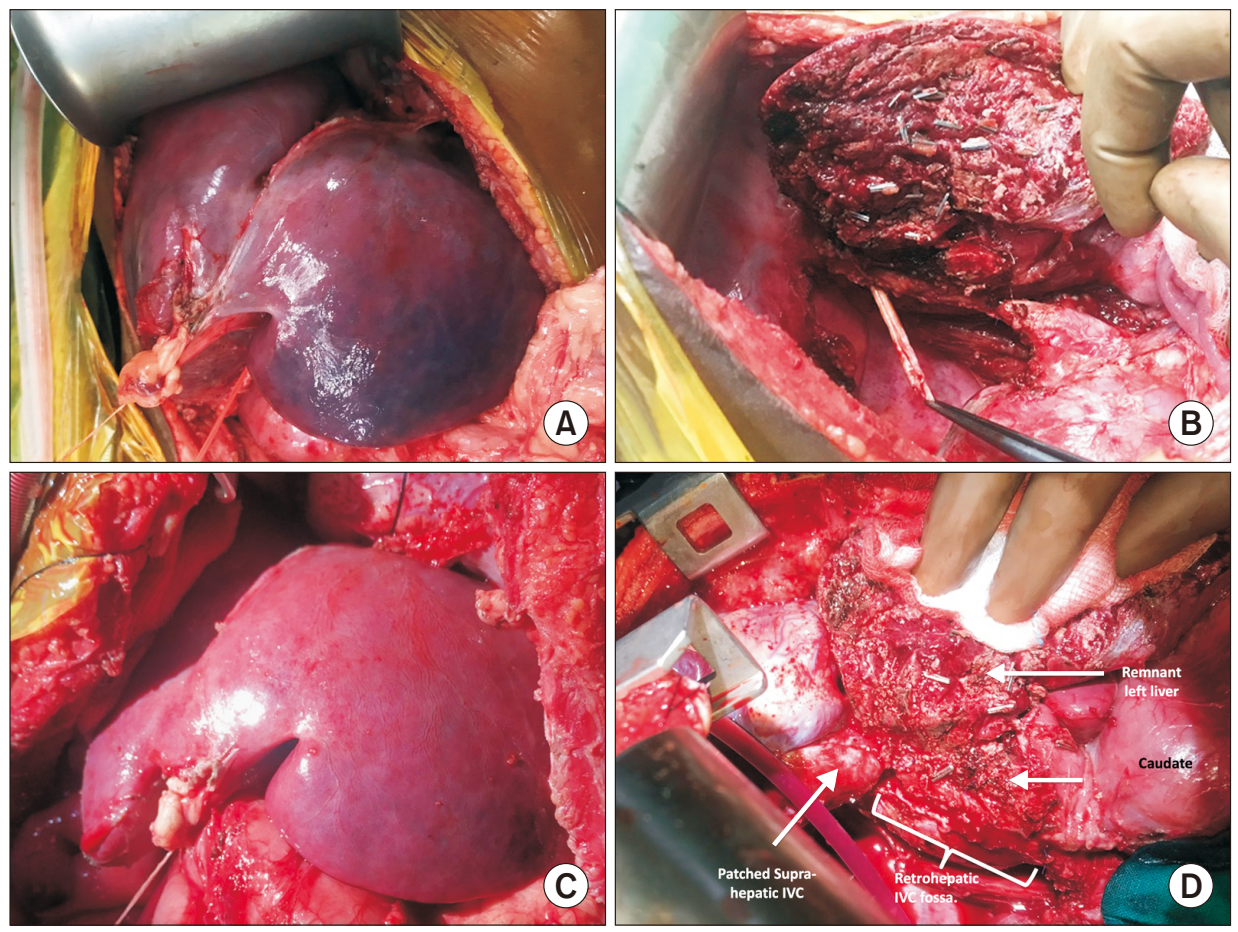

Fig. 2. (A) Congested, dark liver intraoperatively prior to thrombectomy. (B) Raw surface of liver after right hepatectomy and retrohepatic inferior vena cava (IVC) thrombus being extracted with forceps. (C) Near complete resolution of the congestion with pink liver after completion of thrombectomy. (D) At the end of operation, right atrium being closed primarily, IVC at the level of hepatic cloaca being patched with pericardium, and retrohepatic IVC being removed without reconstruction.

primarily. The liver was fixed to the abdominal wall such that the HV remained patent.

The patient tolerated the procedure well. The surgery lasted for 390 minutes with $700 \mathrm{~mL}$ of blood loss. The child had an intensive care stay for 3 days. Sterile abdominal and thoracic collections were found. They were drained without consequence. She was subsequently discharged on postoperative day 11. Hepatectomy specimen measured $13 \mathrm{~cm}$ with a tumor of $4.5 \mathrm{~cm}$ infiltrating the liver and a thrombus of $7.5 \mathrm{~cm}$ in length with an adherent endocardium (Fig. 3). Final histopathology revealed viable residual yolk sac tumor amounting to $1 \%$. The thrombus also showed viable tumor cells without infiltration of the endocardium or the IVC. All margins were microscopically free. The child received three more cycles of PEB. She has been disease-free and symptom-free for the past 12 months with normal AFP levels and normal flows in the HVs on Doppler ultrasound.

Written consent was obtained from patients and/or guardians for publication of clinical and operative details for educational purposes.

\section{DISCUSSION}

IVC thrombosis with RA extension is not uncommonly seen in renal and supra-renal tumors. However, such extensive involvement by GCT is extremely infrequent. Only a few cases have been reported in the literature. Husband and Bellamy [2] have found radiologically detected invasion of IVC in $0.3 \%$ of 397 patients with retroperitoneal disease from GCTs. Savarese

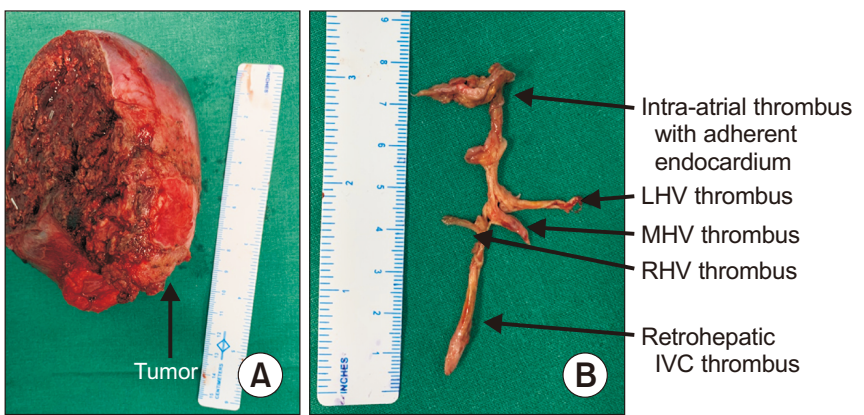

Fig. 3. (A) Right hepatectomy specimen with attached tumor at the inferior surface (arrow). (B) Specimen of the tumor thrombus in the retrohepatic IVC, hepatic veins, and right atrium with adherent endocardium. LHV, left hepatic vein; MHV, middle hepatic vein; RHV, right hepatic vein; IVC, inferior vena cava.

et al. [3] and Miyake et al. [4] have reported two cases of adult GCT with IVC and RA tumor thrombosis managed surgically with cardiopulmonary bypass (CPB). Another testicular GCT recurrence with IVC, RA, and RHV thrombosis has been described in a patient managed with right hepatectomy, cavotomy, and thrombectomy [5]. No account of such extensive vascular thrombus in pediatric GCT could be found in literature. Our report appears to be the first one.

Supra-hepatic IVC and HV thrombosis can lead to eponymous Budd-Chiari syndrome (BCS). The index patient essentially had a type IV BCS [6] with tumor thrombosis as a cause. The only treatment was surgical thrombectomy. This provides 
both symptomatic improvements and potential for long-term survival. Despite responses of the primary tumor and metastatic sites to chemotherapy, more often than not, tumor thrombus persists and requires surgery as in the present case. Our patient had preserved liver functions due to partial flow through the recanalized thrombus as well as collateral pathways via the pericardiophrenic and vertebro-lumbar-azygous veins. Yet, hepatic congestion was intensely discernable both radiologically and intraoperatively. Since the infra-diaphragmatic IVC was completely occluded, our patient tolerated IVC resection without the need for a reconstruction of the entire length. The hepatic cloaca was patched for wide drainage into the residual IVC. Occlusion of all major HVs has been considered either as criteria for inoperability or as candidate for liver transplantation depending on the cause of occlusion while shunting is reserved for those with benign causes of BCS. The present case is the first to demonstrate surgical thrombectomy from all $3 \mathrm{HVs}$ for tumor thrombi.

This case highlights the role of radical surgical resection even in a metastatic disease when the tumor biology is favorable as evidenced by a response to chemotherapy. Notwithstanding an extensive disease that would otherwise be deemed unresectable, complete resections are possible at experienced centers. Cardiac surgical backup is essential since thrombi in the RA usually require $\mathrm{CPB}$ and those isolated to $\mathrm{HVs}$ require a veno-venous bypass. Our large experience of surgical management of tumor thrombi in the IVC and RA from Wilms tumor has allowed us to successfully deal with new challenges of hepatic venous thrombectomy [7]. The other learning point is that pediatric solid tumors are relatively rare. Their referral to specialized tertiary centers is ideal. Administration of chemotherapy without adequate staging workup or multi-disciplinary discussion extenuate the opportunity for correct diagnosis and treatment as in the present case [1].

In conclusion, thrombectomy of all major HVs is feasible with adequate surgical expertise. Their involvement alone should not preclude resection for tumors with a favorable biology. All attempts should be made at surgical thrombectomy as hepatic venous outflow obstruction secondary to tumor thrombosis have no other options that can provide durable symptom and cancer control.

\section{CONFLICT OF INTEREST}

No potential conflict of interest relevant to this article was reported.

\section{ORCID}

Mufaddal Kazi, https://orcid.org/0000-0002-3295-0006

Pradeep Kaushik, https://orcid.org/0000-0001-5177-1022

Shivpal Saini, https://orcid.org/0000-0003-1103-6925

Dhiraj Premchandani, https://orcid.org/0000-0002-4722-375X

Sajid Shafique Qureshi, https://orcid.org/0000-0002-6770-5887

\section{AUTHOR CONTRIBUTIONS}

Conceptualization: MK, PK, SSQ. Data curation: SS, DP, SSQ. Methodology: MK, PK, SSQ. Visualization: MK, SSQ. Writing original draft: MK, SS, DP, SSQ. Writing - review \& editing: MK, SSQ.

\section{REFERENCES}

1. Saini S, Kazi M, Qureshi SS. Miserable 9 years of life: a misdiagnosed and mismanaged case of large retroperitoneal teratoma. Indian J Surg Oncol 2020;11(Suppl 2):253-256.

2. Husband JE, Bellamy EA. Unusual thoracoabdominal sites of metastases in testicular tumors. AJR Am J Roentgenol 1985;145:1165-1171.

3. Savarese DM, Rohrer MJ, Pezzella AT, Davidoff A, Fraire AE, Menon M. Successful management of intracardiac extension of tumor thrombus in a patient with advanced nonseminomatous germ cell testicular cancer. Urology 1995;46:883-887.

4. Miyake M, Fujimoto K, Matsushita C, Chihara Y, Tanaka M, Hirayama A, et al. [Tumor thrombus arising from the superior vena cava and extending into the right atrium in a patient with advanced testicular germ cell tumor]. Hinyokika Kiyo 2009;55:371-375. Japanese.

5. Ní Leidhin C, Redmond CE, Cahalane AM, Heneghan HM, Motyer R, Ryan ER, et al. Radical resection of a late-relapsed testicular germ cell tumour: hepatectomy, cavotomy, and thrombectomy. Case Rep Surg 2014;2014:713049.

6. Martin L, Dondelinger RF, Trotteur G. Treatment of Budd-Chiari syndrome by metallic stent as a bridge to liver transplantation. Cardiovasc Intervent Radiol 1995;18:196-199.

7. Qureshi SS, Bhagat M, Smriti V, Murli D, Baheti A, Yadav S, et al. Intravascular extension of Wilms tumor: characteristics of tumor thrombus and their impact on outcomes. J Pediatr Urol 2021;17:69.e169.e8. 